\title{
Disease activity after the discontinuation of biological therapy in inflammatory rheumatic diseases
}

\author{
Gabriella Kádár • Erika Balázs • Boglárka Soós • \\ Anita Laduver • Péter Keszthelyi • Zoltán Szekanecz • \\ László Kovács
}

Received: 14 May 2013 / Revised: 27 December 2013 / Accepted: 19 January 2014

(C) Clinical Rheumatology 2014

\begin{abstract}
The objectives of this study are to explore the causes of permanent discontinuation of biological therapies in inflammatory rheumatic diseases and to analyse the subsequent course of the disease activity. In this multi-centre retrospective cohort study, data on 126 rheumatoid arthritis, 38 ankylosing spondylitis and 11 psoriatic arthritis patients were analysed, in whom biological therapies had been permanently discontinued. The reasons for the cessation of biologics, the DAS28 or BASDAI disease activity indices at the time of discontinuation and thereafter, and the subsequent occurrence of relapses and the duration of remission or low disease activity were investigated. The most common causes of discontinuation were adverse events (45\%), inefficacy (16\%) or remission (10\%). In rheumatoid arthritis, $33.3 \%$ remained in low disease activity after a mean follow-up of 22 months. If the biologic was stopped when the disease was inactive, $60.6 \%$ remained inactive, and in all the patients in whom the biologic was discontinued because of long-standing remission, the disease remained inactive. Predictors of remission after discontinuation were low disease activity at stopping the biologic and shorter duration of biological therapy. In contrast, $50 \%$ of the ankylosing spondylitis patients relapsed after the withdrawal of anti-TNF therapy. Biologic-free low
\end{abstract}

G. Kádár • E. Balázs • L. Kovács $(\bowtie)$

Department of Rheumatology, Faculty of Medicine, Albert

Szent-Györgyi Health Centre, University of Szeged, Hungary,

Kálvária sgt. 57., Szeged 6725, Hungary

e-mail: kovacs.laszlo@med.u-szeged.hu

B. Soós $\cdot$ Z. Szekanecz

Department of Rheumatology, Institute of Internal Medicine, Medical and Health Sciences Centre, University of Debrecen,

Hungary, Debrecen, Hungary

A. Laduver · P. Keszthelyi

Department of Rheumatology, Pándy Kálmán Békés County

Hospital, Gyula, Hungary disease activity can be achieved in at least one third of rheumatoid arthritis patients, and low disease activity at the time of discontinuation is a strong predictor of a subsequent favourable disease course. The likelihood of continued remission after the cessation of a biological therapy is much lower in ankylosing spondylitis.

Keywords Ankylosing spondylitis · Biological therapy · Discontinuation · Low disease activity $\cdot$ Psoriatic arthritis . Rheumatoid arthritis

\section{Introduction}

The introduction of biological therapies including tumour necrosis factor- $\alpha$ (TNF- $\alpha$ ) inhibitors, interleukin-6 receptor blockers, B cell-depleting antibodies and costimulation blockers into the treatment of inflammatory rheumatic diseases in recent years has led to a complete renewal of treatment goals and therapeutic paradigms. The indications of the initiation of biological therapies have been thoroughly characterised and have been implemented in international and national practice guidelines [1]. However, with the rapidly increasing number of patients on biological therapies and of those who have achieved a long-term remission or low disease activity (LDA) with these agents, the issues of how long biologics should be administered and whether the discontinuation of these drugs can be achieved have gained increasing attention [2]. Potential concerns about the adverse effects and the high costs of long treatment courses also necessitate precise answers to these questions.

The 2010 European League Against Rheumatism (EULAR) recommendations about the treatment of rheumatoid arthritis (RA) suggest that once a sustained remission has been achieved, first the glucocorticoid therapy should be tapered off, and the discontinuation of a biologic disease- 
modifying anti-rheumatic drug (DMARD) may subsequently be attempted. However, these guidelines have not yet been corroborated by firm evidence. The risk of flares after the cessation of an effective therapy and the uncertainty of whether these treatments may be re-introduced successfully also need to be elucidated. Few studies that have been specifically designed to measure the disease activity and radiographic progression in RA after the discontinuation of biologic DMARDs have suggested that long-term biologic-free or even drug-free remission may be achieved in some patients [3-9]. However, predictors of a sustained remission have not been conclusively identified, and the proportions of these patients are variable across the studies; furthermore, only very limited knowledge exists about the disease course after the discontinuation of anti-TNF therapy in ankylosing spondylitis (AS) $[10,11]$ and psoriatic arthritis (PsA).

In this retrospective, multi-centre observational study, our aims were to explore the causes of discontinuation of biological therapies in RA, AS and PsA in the practice of three arthritis centres in Hungary and to analyse the subsequent disease course. We assessed the frequencies of relapses, the duration of remission or LDA and attempted to identify predictors of a sustained remission after the cessation of biologics.

\section{Methods}

The studied patient population consisted of RA, AS or PsA patients treated with biological therapies in Szeged, Debrecen and Gyula, three tertiary arthritis referral centres in southern and eastern Hungary, together serving for a population of approximately 1.5 million people. The protocol was approved by the appropriate ethics committee and has therefore been performed in accordance with the ethical standards laid down in the 1964 Declaration of Helsinki and its later amendments. All patients had been treated with biological therapies (antiTNF, the IL-6 receptor blocker tocilizumab or the anti-CD20 monoclonal antibody rituximab) in accordance with the guidelines by the Hungarian Board of Rheumatologists, which is an adaptation of the EULAR recommendations. RA and peripheral PsA patients had failed a combination of two traditional DMARDs, and AS and axial PsA patients had a highly active disease despite the treatment with at least two non-steroidal anti-inflammatory agents before biologics were initiated. Patients were enrolled in the present investigation if the treating physician decided that the administration of biological therapy is discontinued permanently or until an indefinite time. Onehundred twenty-six patients with RA, 38 with AS and 11 with PsA met these criteria, making a total of 175 patients for the assessment of the causes of discontinuation. These patients constitute $13.7 \%$ (RA), $9.3 \%$ (AS) and $11.2 \%$ (PsA) of the total cohort of biologic-treated patients, respectively. The decision of stopping the biologic was made after a mean of 20 months (RA), 13 months (AS) and 9 months (PsA) of treatment.

The mean age of the patients was 60 years (21-84) in RA, 46 years (20-67) in AS and 57 years (24-74) in PsA. Sixtyeight percent of the RA patients were anti-citrullinated peptide (ACPA) positive, $66.9 \%$ were rheumatoid factor positive, and radiographic erosions were detected in $58 \%$. The causes of discontinuation were categorised as adverse event or allergic reaction (severe or repeated), chronic or recurrent infections, the presence or the suspicion of tumour, long-term remission, family planning and inefficacy or insufficient compliance.

After the cessation of the biological therapy, follow-up data were available in $81 \mathrm{RA}, 17 \mathrm{AS}$ and 2 PsA patients. Follow-up data were, therefore, evaluated only in RA and AS (a total of 98 patients). In RA, we considered the disease of low activity when Disease Activity Score 28 (DAS28) is $<3.2$, whereas high activity was defined as DAS28 $>5.1$. Although remission (DAS28<2.6) has been attained by many of the patients, as the patient population consisted mostly of those with established disease - as it is expected in the routine clinical practice, LDA was used in the statistical analyses. In AS, LDA was defined as Bath Ankylosing Spondylitis Disease Activity Index (BASDAI) of $\leq 2.0$, and a BASDAI $>4$ indicated a high disease activity. The patient was regarded to be in LDA if the disease activity remained low on at least two subsequent visits, while a relapse was considered when the disease activity became high from low or moderate activity at the previous visit. The number of previous biologics used and the corticosteroid and traditional DMARD therapy were recorded in every patient.

The statistical difference between various patient subgroups, including those with relapse after the discontinuation of the biologic or without, was calculated with two-sample $t$ test or chi-square test, depending on whether continuous or categorical variables were assessed. Correlations between certain parameters, e.g. the duration of LDA, and continuous variables (e.g. age, duration of the disease or the biological therapy, DAS28, etc.) were analysed with Pearson's test. The data are presented as mean $\pm \mathrm{SD}$ or mean (range) as appropriate, and a $p<0.05$ value was considered statistically significant.

\section{Results}

The reasons for the discontinuation of the biological therapy are depicted in Fig. 1. As it can be seen, in 78 cases (45\%), the decision was necessitated by significant adverse events or the emergence of contraindications that were deemed by the treating physician to preclude the further use of these types of immunosuppressive therapies. In further 45 cases (30\%), the decision of stopping the biologic was made in agreement 


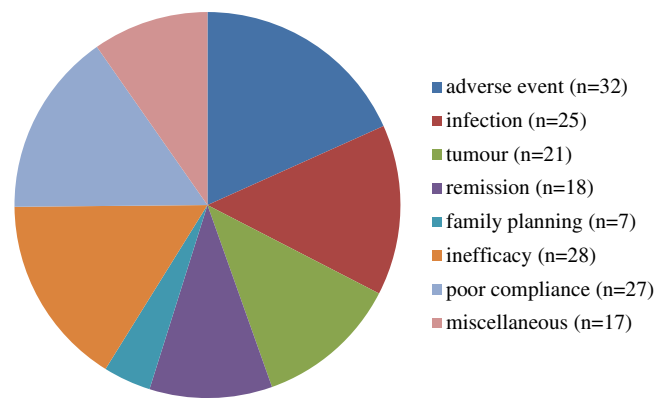

Fig. 1 The distribution of the causes leading to discontinuation of biological therapy. More precise definition of the causes can be found in the "Methods" section

with the patient because of long-standing remission and family planning or when the desired benefits of the treatment were not achieved (inefficacy). Most of these latter cases had been treated with multiple biologics, and in some of them (especially in AS patients), the origin of the complaint has been reconsidered in the light of the clinical course. In the remaining patients, the reason for the cessation was poor compliance or miscellaneous other causes.

In the follow-up population, the data on the biological therapy are to be seen in Table 1. Most of the patients had established RA or AS, and the biological therapy had been administered for several months or years in the majority of the cohort. Switch from one agent to another was common. At the time of the discontinuation, the disease activity was low or moderate in the great majority of the RA patients, whereas it was high or moderate in most of the AS patients.

In the RA patients, after a mean follow-up of 22 months, the disease activity was found to be low in 27 patients $(33.3 \%)$, moderate in $37(45.7 \%)$ and high in the remaining $17(20.9 \%)$ at the time of the analysis (Fig. 2). Twenty of the 33 patients $(60.6 \%)$ in whom the biologic was stopped during LDA have maintained this low activity for an average of 16 months (3-48). Relapse, defined as the increase of disease activity to DAS28 $>5.1$, occurred only in five patients
(15.1\%) after an average of 15 months after the cessation. Consequently, RA was of moderate activity in the remaining patients. The 20 patients with sustained low disease activity are on the following medication: traditional DMARD monotherapy (50\%), low-dose corticosteroid monotherapy (20\%), the combination of the two $(20 \%)$ and no therapy at all $(10 \%)$.

We then sought for the identification of potential associates of sustained LDA. The duration of LDA displayed an inverse correlation with the disease activity measured at the time of the stopping of biologics and - somewhat surprisingly - also with the duration of the biological therapy $(p<0.05)$. Similarly, the occurrence of a relapse was associated with the disease activity at cessation: in those without relapse, the DAS28 was $3.3 \pm 1.3$ in contrast to $4.2 \pm 1.1$ in the relapsers $(p<0.05)$, whereas there was a relationship of borderline statistical significance between the duration of the biological treatment (18.5 \pm 16.1 months in the non-relapsers vs $26.3 \pm 19.3$ months in the relapsers, $p=0.08$ ). In contrast, we have not found any association between the occurrence of a relapse or the duration of LDA and the age of the patients, the ACPA or RF status, the DMARD or corticosteroid treatment after the discontinuation and the reason for the stopping of the biologic, and we also failed to demonstrate an association with the time between the diagnosis of RA and the initiation of biological therapy (i.e. "early" vs "delayed" initiation of the biologic).

In six RA patients, the cessation of the biological therapy was specifically decided because of long-term remission. In all of the patients, the remission has remained sustained during the whole follow-up: its duration is 17,37 and 55 months in three patients who take traditional DMARDs, 7 and 19 months in two patients on traditional DMARDs and low-dose corticosteroid and 3 months in one patient who is completely drug free.

In the AS patients, only three (17.6\%) had inactive disease at the time of the discontinuation of the biological therapy, whereas in five (29.4\%), disease activity was moderate, and
Table 1 Characteristics of the patients in the follow-up cohort

For the definition of disease activity ranges refer to the "Methods" section. Data are expressed as mean (range) unless otherwise indicated

$L D A$ low disease activity

\begin{tabular}{|c|c|c|}
\hline & $\begin{array}{l}\text { RA patients } \\
(n=81)\end{array}$ & $\begin{array}{l}\text { AS patients } \\
(n=17)\end{array}$ \\
\hline Disease duration since diagnosis (months) & $180(32-612)$ & $186(9-396)$ \\
\hline Duration of the biological therapy (months) & $20(1-60)$ & $13(0.5-39)$ \\
\hline Percentage of patients with at least one switch of biologics & 44.4 & 41.1 \\
\hline $\begin{array}{l}\text { Disease activity at the time of discontinuation } \\
\text { (DAS28 for RA and BASDAI for AS) }\end{array}$ & $3.76(1.5-6.8)$ & $4.05(1.0-8.7)$ \\
\hline $\begin{array}{l}\text { Percentages of patients with LDA at the time of the } \\
\text { discontinuation }\end{array}$ & 40.7 & 17.6 \\
\hline $\begin{array}{l}\text { Percentages of patients with moderate disease activity at the } \\
\text { time of the discontinuation }\end{array}$ & 52.1 & 29.4 \\
\hline $\begin{array}{l}\text { Percentages of patients with high disease activity at the time } \\
\text { of the discontinuation }\end{array}$ & 17.2 & 52.9 \\
\hline
\end{tabular}




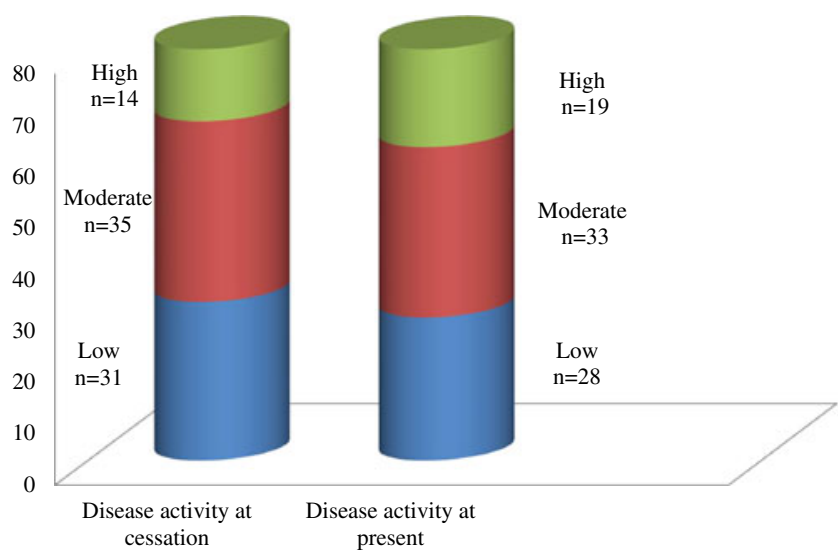

Fig. 2 The change in the numbers of rheumatoid arthritis patients with high, moderate or low disease activity after the discontinuation of the biological therapy

in nine of them (52.9\%), AS was active. After the subsequent follow-up of an average of 20 months, the percentages of patients with inactive, moderately active and active disease have become $11.7 \%(n=2), 35.2 \%(n=6)$ and $52.9 \%(n=9)$, respectively. Relapse occurred in four of the eight patients in whom the biologic had been stopped at BASDAI $<4.0$ after a mean of 6 months (3-9). The two patients who are currently in remission (BASDAI $<2.0$ ) have had an inactive disease for 41 and 2 months. After the stopping of the biologics, sulfasalazine has been administered to two patients, while the remaining patients do not receive any DMARD. Probably due to the low number of patients in remission, we failed to identify any factor that may be predictive of a favourable disease course after the cessation of the biological therapy.

\section{Discussion}

Important issues of rheumatology currently and in the near future are to more precisely determine the optimal duration of biological therapies, to identify the causes that lead to the discontinuation of these treatments and to explore the predictors of a favourable disease outcome after the stopping of biologics, i.e. long-term remission without functional and radiographic progression. As our knowledge in these questions is limited, this study may add useful information about the natural course of RA, AS and PsA from the daily clinical practice to the results of prospective studies with fixed protocols. It is important that the three centres that participate in this project strictly adhere to the current European guidelines for the treatment of inflammatory arthritides and follow similar treatment strategies.

After an average treatment duration of 20 months (RA patients) or 13 months (AS patients), in $13.7 \%$ and $9.3 \%$ of the patients, respectively, biologics were permanently discontinued. The most common causes of the cessation were adverse events that were considered by the treating physician to have a high likelihood of recurrence and to have a chronic course, or the re-introduction of the same or other biologic would be associated with inacceptable risks. The second largest subgroup comprised patients in whom the biologic was deliberately stopped after the joint decision of the physician and the patients. In $10 \%$ of the patients, long-term remission was the reason of the cessation, and although this is a significant proportion of the cohort, it reflects that a decision of stopping the biological therapies in patients with longstanding remission is not yet part of the routine clinical practice, similar to the international trends.

Our follow-up results indicate that the LDA is feasible in a significant proportion of patients: more than $30 \%$ of those with RA had inactive disease at an average of 22 months after the biological therapy had been stopped. In AS, however, the risk of the recurrence of high disease activity is much higher, and only $11.7 \%$ of the patients remained in remission. RA patients had a higher chance of maintaining the LDA and a lower risk of a relapse of the disease if they had low DAS28 when the biologic was stopped. As much as $60.6 \%$ of those RA patients who had LDA at discontinuation as compared with $30 \%$ of the whole cohort remained in LDA. These results indicate that considering the discontinuation of a biological therapy may be a realistic option in many of the RA patients who have entered into remission or at least LDA.

Only few prospective studies have addressed the issue of the discontinuation of a biologic treatment. In the first such study, the TNF20 examination, 10 recent-onset RA patients were followed up after the cessation of infliximab therapy administered for 1 year [4]. Nine of these patients went into remission during anti-TNF treatment, and seven of them remained so after a follow-up of 1 year. The largest study in this regard so far, the BeSt trial, has follow-up data of more than 5 years available [5]. This study compared four treatment strategies with a strict dose escalation and tapering protocol. Of the 120 patients who were randomized to receive infliximab + methotrexate, 77 achieved a sustained remission, and infliximab was subsequently withdrawn. In 43 patients $(56 \%)$, remission has persisted thereafter during an average of 7.2 years of follow-up [5] without radiographic progression for several years [6]. In the OPTIMA study, early RA patients (average symptom duration, 3.9 months) had been treated with adalimumab + methotrexate and were subsequently randomized to receive either methotrexate+ placebo or methotrexate+adalimumab [7]. In $66 \%$ of those in whom the adalimumab had been withdrawn, the remission persisted on methotrexate monotherapy. In addition to these studies on RA patients with short disease duration, two studies included patients with established RA: the RRR trial results revealed that $55 \%$ of the patients preserved the LDA after 1 year after 
the cessation of infliximab [8], whereas interim analysis of the HONOR examination indicates that $73 \%$ of the patients remained in LDA 6 months after the discontinuation of adalimumab [9]. Factors that were suitable to predict the maintenance of LDA or remission were low disease activity at cessation (HONOR, RRR), less functional impairment as reflected by a low HAQ-DI index (OPTIMA), shorter disease duration before the initiation of anti-TNF therapy (RRR, BeSt, Chimenti et al. [10]), younger age (RRR), less radiographic damage (RRR) and the absence of ACPA (BeSt). Our results confirm the importance of maintaining a low disease activity before the cessation of biologics could be considered.

Another feature that has proven to be significantly associated with a subsequent relapse-free disease course in our study was a shorter duration of biological therapy. This seems surprising, but one potential explanation is that these patients may have a relatively milder disease that had responded better or faster to biological therapy than those who needed longer biological treatment and still relapsed after the cessation. The previously cited studies also indicate that 6 or 12 months of anti-TNF treatment is sufficient for the appropriate patients to maintain the LDA after its cessation, provided that it had resulted in an effective control of the disease.

We have even less evidence regarding the biologic-free disease course in AS, but our experience is in accord with the published results in that it is much less likely for an AS patient to remain in inactive disease after an anti-TNF treatment is stopped. In the study of Baraliakos et al., $90.5 \%$ of the patients relapsed until the 36 th week after the withdrawal of the biologic, and in $24 \%$, the relapse has occurred as early as before the 12th week [11]. Brandt et al. have found that all the AS patients whose etanercept therapy had resulted in complete remission have relapsed by the 24th week after its withdrawal [12]. It is not known at present whether a subgroup of AS patients may exist in whom the long-term withdrawal of antiTNF therapy may be feasible, and some investigators have, therefore, aimed at examining the effects of dose reduction instead of complete drug withdrawal, with promising results [13], although this strategy is also far from being well explored.

In summary, with a goal-steered therapeutic strategy, the discontinuation of biological therapies is likely to be possible in a relatively large proportion of RA patients. Our results and others indicate that an established remission is a pre-requisite for the successful withdrawal of the biologics; furthermore, earlier diagnosis and treatment initiation, and a resulting better functional status and less radiographic damage also carry a better prognosis of a biologic-free remission.
Acknowledgments The work was supported by EU grants TÁMOP4.2.2.A-11/1/KONV-2012-0035 and TÁMOP-4.2.2/B-10/1-2010-0012.

Disclosures None.

\section{References}

1. Smolen JS, Robert Landewé R, Breedveld FC et al (2010) EULAR recommendations for the management of rheumatoid arthritis with synthetic and biological disease-modifying antirheumatic drugs. Ann Rheum Dis 69(6):964-975

2. Saleem B, Keen H, Goeb Vet al (2010) Patients with RA in remission on TNF blockers: when and in whom can TNF blocker therapy be stopped? Ann Rheum Dis 69:1636-1642

3. Goekoop-Ruiterman YP, de Vries-Bouwstra JK, Allaart CF et al (2008) Clinical and radiographic outcomes of four different treatment strategies in patients with early rheumatoid arthritis (the BeSt study): a randomized, controlled trial. Arthritis Rheum 58(2 Suppl):S126-S135

4. Quinn MA, Conaghan PG, O'Connor PJ et al (2005) Very early treatment with infliximab in addition to methotrexate in early, poorprognosis rheumatoid arthritis reduces magnetic resonance imaging evidence of synovitis and damage, with sustained benefit after infliximab withdrawal: results from a twelve-month randomized, double-blind, placebo controlled trial. Arthritis Rheum 52:27-35

5. van den Broek M, Klarenbeek NB, Dirven L et al (2011) Discontinuation of infliximab and potential predictors of persistent low disease activity in patients with early rheumatoid arthritis and disease activity score-steered therapy: subanalysis of the BeSt study. Ann Rheum Dis 70:1389-1394

6. van der Kooij SM, Goekoop-Ruiterman YP, de Vries-Bouwstra JK et al (2009) Drug-free remission, functioning and radiographic damage after 4 years of response-driven treatment in patients with recentonset rheumatoid arthritis. Ann Rheum Dis 68:914-921

7. Kavanaugh A, Emery P, Fleischmann R, et al (2011) Withdrawal of adalimumab in early rheumatoid arthritis patients who attained stable low disease activity with adalimumab plus methotrexate: results of a phase 4 , double-bind, placebo-controlled trial. ACR 2011, abstract No 1699

8. Tanaka Y, Takeuchi T, Mimori T et al (2010) Discontinuation of infliximab after attaining low disease activity in patients with rheumatoid arthritis: RRR (remission induction by Remicade in RA) study. Ann Rheum Dis 69:1286-1291

9. Tanaka Y, Hirata S, Nawate M, et al (2011) Discontinuation of adalimumab without functional and structural progress after attaining remission in patients with rheumatoid arthritis (an interim report of HONOR study). ACR 2011 abstract No 2468

10. Chimenti MS, Graceffa D, Perricone R (2011) Anti-TNF $\alpha$ discontinuation in rheumatoid and psoriatic arthritis: is it possible after disease remission? Autoimmun Rev 10:636-640

11. Baraliakos X, Listing J, Brandt J et al (2005) Clinical response to discontinuation of anti-TNF therapy in patients with ankylosing spondylitis after 3 years of continuous treatment with infliximab. Arthritis Res Ther 7:R439-R444

12. Brandt J, Khariouzou A, Listing J et al (2003) Six-month results of a double-blind, placebo-controlled trial of etanercept treatment in patients with active ankylosing spondylitis. Arthritis Rheum 44:342-3484

13. Navarro-Compán V, Moreira V, Ariza-Ariza R et al (2011) Low doses of etanercept can be effective in ankylosing spondylitis patients who achieve remission of the disease. Clin Rheumatol 30:993-996 